\title{
The Design of the High Voltage Switch Detection System of the Power System and Research
}

\author{
Yang Gao ${ }^{1, a^{*}}$, Xuemin Leng ${ }^{1, ~ a ~}$, Wei Zheng ${ }^{2, b}$, Xiaoming Feng ${ }^{2, b}$, Aoran $\mathrm{Xu}^{1 \text {, a }}$ \\ ${ }^{1}$ Shenyang Institute of Engineering, Shenyang City, Liaoning Province, China \\ ${ }^{2}$ Yingkou power supply company, Liaoning Province, China State Grid Corp \\ aemail: gaoyangsie@163.com
}

Keywords: high voltage switch; mechanical characteristics; on-line monitoring; force sensor.

Abstract: Study of on-line monitoring of high voltage circuit breaker has important economic significance and social significance to improve the maintenance level of power system. According to the characteristics of the circuit breaker's contact system and pull arm structures, a specific force sensor was designed, as well as its signal processing circuit. And then all experimental platform on the mechanical characteristics of the circuit breaker was set up, which could measure the main circuit parameters and the force sensor output signal. Moreover, this platform could not only realize the conventional off-line measurement systems for the mechanical characteristics, but also the on-line ones. According to test results, the previous simulation were validated, namely, the correspondence between the instantaneous opening (closing) moment and the mutation force signal in the pull of such circuit breaker.

\section{Introduction}

The circuit breaker is an essential device which plays an important role in power grid scheduling, power system controlling and protective relaying. Due to the complexity of mechanical structure, the failure probability of the circuit breaker is higher than any other power devices. So the on-line monitoring of circuit breaker mechanical characteristic becomes the important part of the circuit breaker online monitoring. In this paper, in order to study the online monitoring of the circuit breaker mechanical properties, we focus on testing the practical application of the force sensor, and verifying that it can be achieved through force signals to measure mechanical properties of the circuit breaker. We design a circuit breaker mechanical characteristic test system which includes traditional measuring of primary loop make-and-break and the force signal measurement [1-4]. Using this test system, the circuit breaker mechanical properties measurements can be realized in both the traditional way of online and offline of force signal.

\section{Design of experiments}

The development of the test system should be based on the development of the force sensor in order to verify the moment when the rigid on/off obtained online through the force sensor. Since that, the circuit breaker mechanical characteristics test system we designed can make the monitoring system get various data according to measure time of the force sensor.

The principle of achieving the main circuit's measurements of disconnection and closure is showed in figure 1. Injection current of contacts is provided by an external current source, after injection current, the main loop's switch is closed, the voltage of sampling points Tout presents a high level, current reaches the optical coupling switch through R2, optical coupling switch TLP521.4 is a pathway and the output end of $\mathrm{V}$ out shows a high level. When the main loop' switch is turned off, there is no current flowing past the sampling point Tout, exhibiting a low level, optical coupling switch TLP521.4 has no circulation current and the output end is at a low level. However, the acquisition card can get main loop's on-off through collecting the high and low level of the output end, but the main circuit's on-off is the open or closing moment of the contact. 


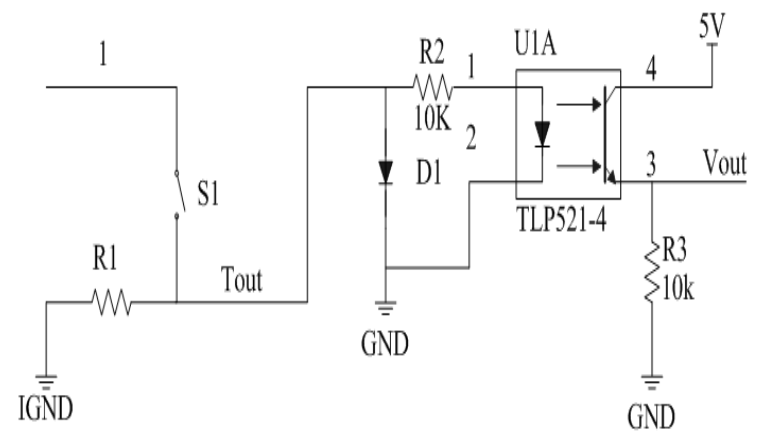

Fig. 1 the principle of on-off measurement of the main circuit.

The measurement of the coil current in the control loop is required for higher precision. In this paper, we use the Holzer current sensor, which can accurately sample the DC, and can accurately track the dynamics of the exchange. Because the Holzer current sensor is a current, voltage converting unit, in order to meet the needs of the monitoring coil current, the current sensor is rated at $10 \mathrm{~A}$ to $-10 \mathrm{~A}$. When the peripheral circuit is designed, its input and output characteristics are shown in figure 2:

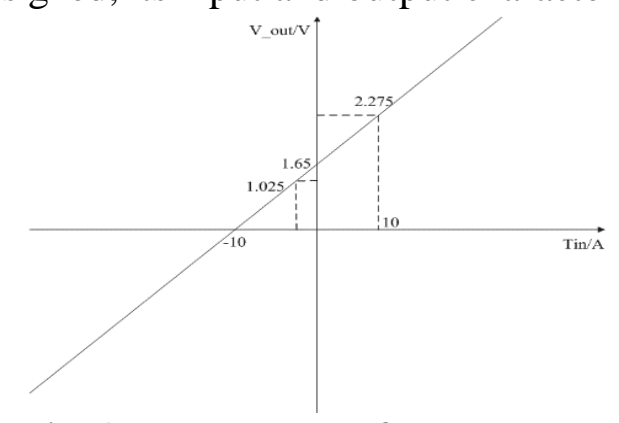

Fig. 2 output curve of current sensor

The current sensor will be linear transformation of the current and voltage, the input current linear transform into voltage, the rated input voltage is from $-10 \mathrm{~A}$ to $10 \mathrm{~A}$, the corresponding output is $1.031 \mathrm{~V}-2.269 \mathrm{v}$ and zero input is $1.59 \mathrm{~V}$. The circuit diagram of the current input channel is shown in Figure 3:

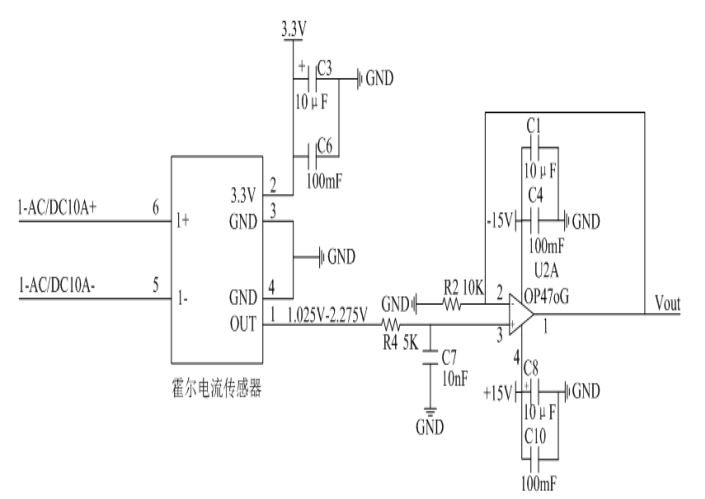

Fig. 3 current sampling and conditioning circuit

Above is the current sampling and conditioning circuit. In order to improve the measuring accuracy of the system and increase the anti-interference ability, that needs to join the emitter follower. Adding an emitter follower with the core component of OP470 before inputting AD can improve the accuracy of the system. Sampling resistor in the system uses film resistor, metal precision is $0.1 \%$. The decoupling capacitor is arranged on the power input point of the Holzer current sensor and the emitter follower circuit. The decoupling capacitor is composed of an electrolytic capacitor and a ceramic capacitor. The capacitor is selected for $10 \mathrm{uF}$, and the ceramic capacitor is selected for $100 \mathrm{nF}$, it can't be ignored the influences of the capacitance frequency characteristic, leakage current, capacitance value, volume and so on. At the same time, the process of the acquisition of signal is often affected by the noise and the environment, so the reduction of real signal needs to use the filter. 


\section{Software Development}

PC has functions, including the acquisition card of analog quantity acquisition, the closing time of the calculation, the tripping and closing coil current, displacement and tensile force of collection and so on. The following focus on how to achieve the acquisition card collection procedures. Data acquisition procedures for the use of the development process are $\mathrm{C}++\mathrm{Builder}$, which is provided by the data acquisition card is API development kits.

The following is detailed flow chart:

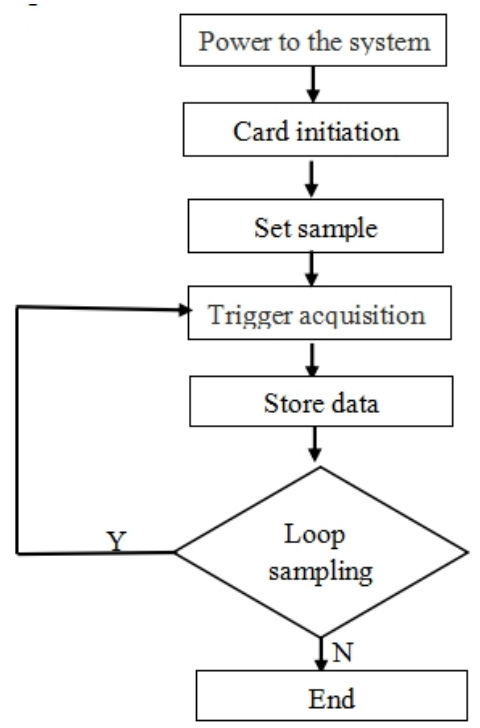

Fig. 4 data acquisition process

The acquisition process is as follows: after the system is powered up, the board is initialized and the number of available channels is obtained. Then make settings related data acquisition, including the number of channels to set up the channel, sampling frequency, through the software to trigger the start of data acquisition, the acquisition of the data stored in the file system. According to the demand to determine whether to do a number of repeated sampling, loop sampling is achieved through the timer function.

\section{Experiment of Testing System}

The test of testing system is the use of development of circuit breaker test system and it tests through closing and tripping the circuit breaker.

According to the experimental results, we can see that the three-phase curve is similar .Taking the A phase as an example, compare and analyze the actual process of the pull force curve and the simulation of the force curve. A pull force test system in the process of circuit breaker closing in the test of and the resulting displacement are shown in figure 5:

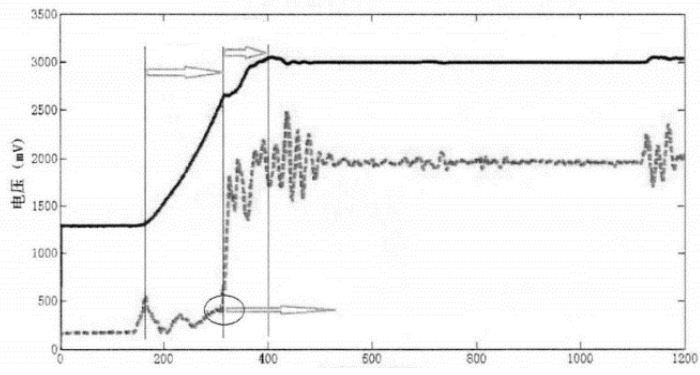

Fig. 5 actual closing process of A rod displacement and pull force

Pull force is produced of the closing action of A phase in the process of simulation testing as shown in Figure 6 : 


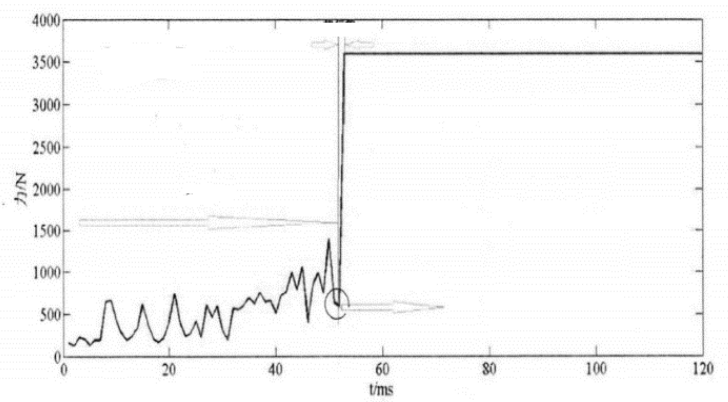

Fig. 6 closing simulation process of A phase pull force

In the simulation test and the actual test, before in the absence of contacting with the hydraulic buffer, the curves and the experimental data's law are in agreement with the two. So according to the experimental curves and the results and compare them to a conclusion that in the closing action, the changeable moment that pull force measuring curve in the stationary wave begins to rise suddenly is the instantaneous moment of closing brake. In the opening action, the transmission mechanism from the moment of beginning to decline until just separating brake in the process of break-brake is the moment of separation.

By analyzing the same points and different points of the actual curve and simulation curve, we calculate the critical moment of breaker and switch and can realize the on-line measurement of the mechanical characteristics of circuit breaker.

\section{Conclusion}

This paper mainly studies how to realize the online monitoring circuit breaker's mechanical proper ties.In view of the existing online measuring circuit breaker mechanical characteristic,there is a probl em that measurement accuracy is not high in related traditional offline,put forward using lever force $s$ ignal points just moment access method. Not only by the results of simulation test to measure accurat ely, and the result is verified by practical experiment to further accurate, practical and simulation bot $\mathrm{h}$ combination represents a combination of online and offline measurement, to two different testing $\mathrm{m}$ ethods can be combined unification has laid a solid foundation platform.In order to achieve the force signal acquisition of reality.

\section{References}

[1] Ann Tao, Qingfeng Wang, Jianzhong Tang, Yao Ming, Application of the circuit breaker operation structure simulation model in fault diagnosis, Advanced Technology of Electrical Engineering and Energy. 29 (2010) 41-43.

[2] Lu Guo, Zhongjian Li, Hao Lei, The design of the high voltage switch mechanical properties testing system, Computer Measurement and Control. 18(2010) 232-233.

[3] Wenji Qin, Lili Ma, Congming Wei, Research on the mechanical properties on-line monitoring of high voltage circuit breaker, Electric Switch. 05 (2012) 51-53.

[4] T. Watanabe, T. Sugimoto, H. Imagawa, C. K. Keong, C. T. Yong, Q. S. Zhen, Practical application of diagnostic method of circuit breaker by measuring three current wave forms, Condition Monitoring and Diagnosis. 11(2008) 398-401.

[5] Jiangtao Sun. Development of the Test System for the Mechanical Characteristics of the circuit breaker based on ATmegal6, Dalian University of Technology, China, 2009. 